Special Issue of the 7th International Advances in Applied Physics and Materials Science (APMAS 2017)

\title{
Performance of Free Space Optical Terrestrial Link under Dense Fog Conditions
}

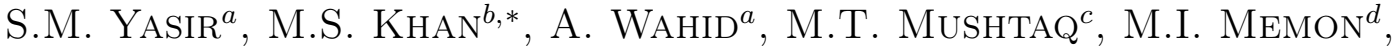 \\ M. SIRAJ ${ }^{e}$ AND M.S. IQBAL ${ }^{f}$ \\ ${ }^{a}$ Department of Electrical Technology, Government Swedish Pakistani Institute of Technology, Gujrat, Pakistan \\ ${ }^{b}$ Department of Electrical Engineering, Faculty of Engineering, Lahore Leads University, Lahore, Pakistan, \\ Lahore Leads University, Lahore, Pakistan \\ ${ }^{c}$ School of Systems and Technology (SST), UMT Lahore, Pakistan \\ ${ }^{d}$ Department of Electronics and Telecommunications Engineering, \\ Global College of Engineering and Technology, Muscat \\ ${ }^{e}$ College of Engineering, King Saud University, Riyadh, Saudi Arabia \\ ${ }^{f}$ Department of Electrical and Electronic Engineering, KOÇ University, Istanbul, Turkey
}

\begin{abstract}
Free-space optical links use modulated beam of light to transmit high amount of data from transmitter to receiver to get line-of-sight communication link. Free-space optical is cost effective solution to provide higher data rate to end-users. Free-space optical links are also considered as a better alternative to RF links due to their advantages of low power consumption and higher data rate of the range Gbps, and are highly secure to electromagnetic interference. Despite of having all these advantages free-space optical links are highly affected by the severe weather conditions like fog, rain, snow, smoke and dust or aerosol particles suspended in air. Fog is one of a major challenge for free-space optical to achieve carrier class availability and causes high amount of attenuation. In this paper we presented the performance analysis of free-space optical links by estimating signal-to-noise ratio, bit error rate, under fog conditions during winter season in Lahore, Pakistan.
\end{abstract}

DOI: 10.12693/APhysPolA.134.268

PACS/topics: FSO, OWC, optical wireless communication, fog, attenuation

\section{Introduction}

Ever increasing demands of bandwidth for telecommunication applications have led the scientists and engineers to develop communication link which has capability to fulfill continually growing demands of bandwidth (or higher data rates) at lowest cost. Radio frequency $(\mathrm{RF})$ communication link conventionally permits limited data rate (10-622 Mbps) due to congested spectrum $[1,2]$. The next generation wireless communication systems should have capability to fulfill the demands of high data rates for broadband global services, significantly increasing mobile users, live streaming, video calling, and conferencing. Optical fiber can be used to meet the needs of higher data rates but it is not an economical option for end users. Free-space optical links (FSO) can be used to provide higher data rates as that of fiber optics. FSO can be used as last mile connectivity because of their benefits of higher data rate, higher mobility and flexibility of link installation and cost effectiveness [3-5]. The FSO communication link is a line of sight communication link which normally uses the intensity modulated or on-off shift keying (OOK) modulated or wavelength division multiplexing laser beam as a communication carrier at terahertz $(\mathrm{THz})$ frequency. The propagating sig-

*corresponding author; e-mail: msaeedbaloch@gmail.com nal travels through atmospheric channel and suffer attenuations from fog, smoke, dust etc. In Pakistan, the FSO link is mostly attenuated due to fog. Usually fog appears in the months of December, January, and February and remained compact for several hours. In this paper we analyze the performance of FSO links under dense fog conditions.

\section{Attenuation due to fog}

Fog reduces the visibility of atmospheric layer, which in turn causes the optical attenuation due to the Mie phenomenon because the size of fog particles are comparable to transmitted optical wavelengths. Reduced visibility causes severe attenuation for FSO links. There are several models widely used to estimate optical attenuation using the reduced visibility and these models are known as Kruse, Kim, and Al-Nabulsi models [6-8]. We collected the visibility data from metrological department, Lahore, Pakistan from Dec, 2014 to Feb, 2015. There are different fog events observed during the mentioned period. The details of observed fog events and estimated optical attenuation is given in Table I.

\section{Design parameters for FSO communication link}

We choose the following design parameters of FSO communication links to improve the link quality and for analysis based on the fact of availability and their usage in commercial systems and are given below in Table II. 
Critical fog events during 17-12-2014 to 10-01-2015.

TABLE I

\begin{tabular}{|c|c|c|c|c|c|c|c|}
\hline \multicolumn{2}{|c|}{ Event start } & \multicolumn{2}{|c|}{ Event ending } & \multirow{2}{*}{$\begin{array}{l}\text { Duration } \\
\text { of event }[\mathrm{h}]\end{array}$} & \multicolumn{3}{|c|}{ Attenuation $[\mathrm{dB} / \mathrm{km}]$} \\
\hline Date & Time & Date & Time & & Max. & Min. & Mean \\
\hline $17-12-2014$ & 12:00am & $18-12-2014$ & 1:00pm & 14 & 551.3 & 9.25 & 239.8 \\
\hline $18-12-2014$ & 9:00am & $19-12-2014$ & 06:00pm & 22 & 28.88 & 9.25 & 16.03 \\
\hline $20-12-2014$ & $11: 00 \mathrm{pm}$ & $21-12-2014$ & 12:00am & 14 & 207.96 & 9.25 & 142.4 \\
\hline $23-12-2014$ & 8:00pm & $24-12-2014$ & 11:00am & 17 & 551.7 & 12.99 & 350.7 \\
\hline $24-12-2014$ & $3: 00 \mathrm{pm}$ & $25-12-2014$ & 12:00am & 9 & 1141 & 9.25 & 316.3 \\
\hline $27-12-2014$ & 7:00am & $28-12-2014$ & 2:00am & 20 & 98.12 & 9.25 & 34.51 \\
\hline $28-12-2014$ & 7:00am & $29-12-2014$ & $1: 00 \mathrm{pm}$ & 14 & 207.9 & 7.25 & 60.12 \\
\hline $29-12-2014$ & 12:00am & $30-12-2014$ & $1: 00 \mathrm{pm}$ & 38 & 1141 & 7.09 & 371.1 \\
\hline 03-01-2015 & $12: 00 \mathrm{pm}$ & 04-01-2015 & $12: 00 \mathrm{pm}$ & 37 & 325.7 & 10.85 & 112.3 \\
\hline 08-01-2015 & $9: 00 \mathrm{pm}$ & 09-01-2015 & $12: 00 \mathrm{pm}$ & 40 & 81.43 & 16.28 & 35.98 \\
\hline 10-01-2015 & 11:00pm & $12-12-2015$ & 12:00pm & 38 & 325.7 & 23.26 & 108.0 \\
\hline
\end{tabular}

Design parameters of FSO link

TABLE II

\begin{tabular}{l|c}
\hline \multicolumn{1}{c|}{$\begin{array}{c}\text { FSO system } \\
\text { design parameters }\end{array}$} & Value \\
\hline wavelength & $1550 \mathrm{~nm}$ \\
transmitted power & 5 to $100 \mathrm{~mW}$ \\
link range & $1 \mathrm{~km}$ \\
diam. of transmitter aperture & $10 \mathrm{~cm}$ \\
diam. of receiver aperture & $15 \mathrm{~cm}$ \\
divergence angle & $1.5 \mathrm{mrad}$ \\
receiver efficiency & $85 \%$ \\
transmitter efficiency & $85 \%$ \\
sensitivity factor & $20 \mathrm{dBm}$ \\
band width & $100 \mathrm{MHz}$ \\
fade margin & $2.5 \mathrm{~dB}$ \\
receiver noise figure & $2.5 \mathrm{~dB}$
\end{tabular}

\subsection{Received power versus optical attenuation}

Attenuation is a loss of optical signal power offered by the randomly appeared atmospheric parameters. The mathematical relationship is stated in the equation below

$$
\text { Power }_{\text {received }}=P_{r x} X\left(\frac{D^{2}}{\theta^{2} X L^{2}}\right) X 10^{-\frac{\gamma L}{10}} \tau_{r x} \tau_{t x},
$$

where $D$ is the receiver diameter, $\theta$ is the divergence angle, $\gamma$ is the attenuation coefficient, $\tau_{t x}$ and $\tau_{r x}$ are the transmitter and receiver optical efficiency, respectively. The relationship between attenuation and received power $(\mathrm{dBm})$ is simulated at different transmitted powers shown in Fig. 1a. Simulation result shows that above $60 \mathrm{~dB} / \mathrm{km}$ attenuation, received power is reduced which limits the link margin and hence the reliability of the FSO system decreases. The maximum received powers at attenuation of $7.09 \mathrm{~dB} / \mathrm{km}$ are $0.059,0.0844,0.0992,0.1098$, and $0.1688 \mathrm{~dB} \mathrm{~m}$ at $5,10,15,20$, and $100 \mathrm{~mW}$ transmitted power levels, respectively.

\subsection{Data rate versus optical attenuation}

Higher amount of received power results in higher data rate, link margin and lower attenuation. Higher attenua- tion will reduce the data rate as shown in Fig. 1b. When optical attenuation is about $7.09 \mathrm{~dB} / \mathrm{km}$, at $5 \mathrm{~mW}$ of transmitted power, the FSO link has capacity to transmit the data of the rate 77.3 Gbps. At the same attenuation level of $7.09 \mathrm{~dB} / \mathrm{km}$, if the transmitted power increases to $10 \mathrm{~mW}$, the data rate will be $158 \mathrm{Gbps}$. Similarly, by increasing transmitted power both under clear sky conditions or under foggy conditions data rate can be improved as shown in Fig. 1b.
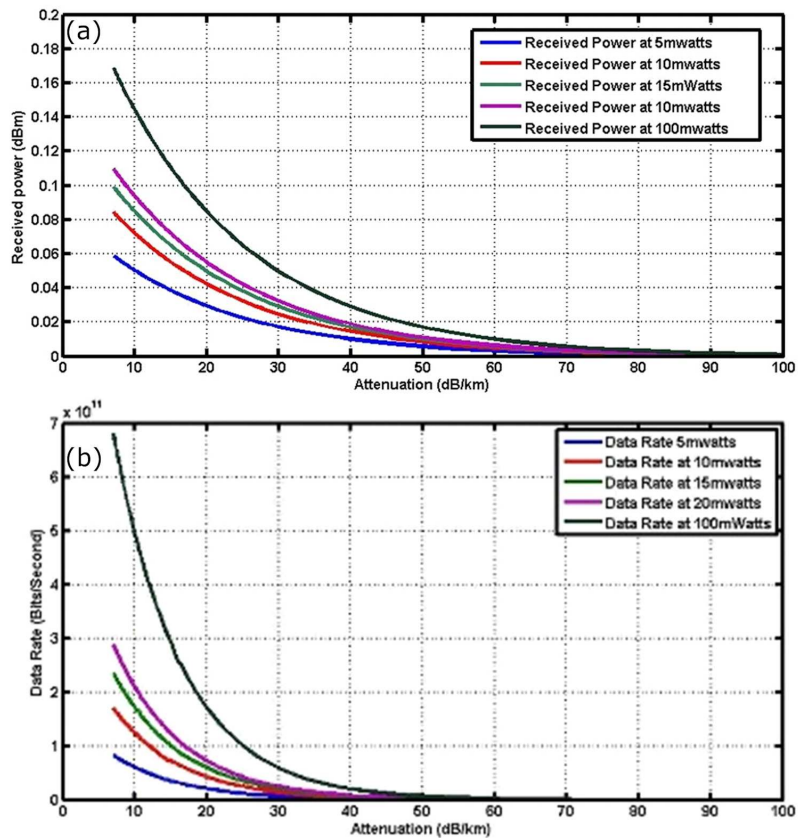

Fig. 1. a) Received power against attenuation, (b) attenuation against data rate.

\subsection{SNR versus attenuation}

Signal-to-noise ratio (SNR) is an important parameter of communication link to determine the link quality and has inverse relationship with attenuation. Figure 2a shows that as attenuation increases SNR decreases but decrease in SNR is not very sharp. The mathematical 
expression of SNR is described in Eq. (2):

$$
\begin{aligned}
& \mathrm{SNR}=P_{T}-30+10 \log \left(G_{T}\right)+10 \log \left(G_{R}\right) \\
& -20 \log \left(\frac{4 \pi}{\lambda}\right)-10 \log \left(k_{B} B_{W} T\right)-\alpha-N_{F}-F_{M},
\end{aligned}
$$

where $P_{T}$ is the transmitted power, $G_{T}$ is the transmitter antenna gain, $G_{R}$ is the receiver antenna gain, $\lambda$ is the optical signal wavelength, $k_{B}$ is the Boltzmann constant, receiver bandwidth $\left(B_{W}=1 \mathrm{MHz}\right), T$ is the ambient temperature in $\mathrm{K}, N_{F}$ is the receiver noise figure, $F_{M}$ is the fade margin, and $\alpha$ is the total attenuation in $\mathrm{dB} / \mathrm{km}$. Antenna gains for both transmitter and receiver can be expressed as given in Eqs. (3) and (4), respectively

$$
\begin{aligned}
G_{T} & =\frac{32}{{\text { (Divergence Angle })^{2}}^{2}} \\
G_{R} & =\left(\frac{\pi D r}{\lambda}\right)^{2} .
\end{aligned}
$$

The SNR increases as the transmitted power increases because SNR is a ratio of signal power and noise power. When signal power is improved by increasing the transmitter power the ratio improves so which can be observed from Table III.

Data statistics of SNR versus attenuation

TABLE III at different transmitter power

\begin{tabular}{l|c|c|c|c|c}
\hline \hline SNR [dB] & $5 \mathrm{~mW}$ & $10 \mathrm{~mW}$ & $15 \mathrm{~mW}$ & $20 \mathrm{~mW}$ & $100 \mathrm{~mW}$ \\
\hline min. SNR & 121.3 & 124.3 & 126.1 & 127.3 & 134.3 \\
max. SNR & 122.4 & 125.5 & 127.2 & 128.5 & 135.5 \\
mean SNR & 122.4 & 125.4 & 127.2 & 128.4 & 135.4 \\
std. SNR & 0.1328 & 0.1328 & 0.1328 & 0.1328 & 0.1328
\end{tabular}

\subsection{BER versus attenuation}

Bit error rate (BER) is another important communication factor which defines the quality and reliability of the link. Higher attenuation will increase BER which is inversely proportional to the SNR. If signal has greater noise power BER will be high and system data reliability will decrease. If BER is high, quality of signal received will be poor. The mathematical relationship is given in Eq. (5) [9]:

$$
\mathrm{BER}=\left(\frac{2}{\pi \mathrm{SNR}}\right) \exp \left(-\frac{\mathrm{SNR}}{8}\right) .
$$

As attenuation increases, BER also increases. This relationship is simulated for $5,10,15,20$, and $100 \mathrm{~mW}$ and shown in Fig. 2b. Maximum value of BER is $1.363 \times 10^{-9}, 9.128 \times 10^{-10}, 7.223 \times 10^{-10}, 6.118 \times 10^{-10}$ and $2.421 \times 10^{-10}$ at respective transmitted powers.

\section{Conclusions}

It is observed that increase in transmit power (within limitation) is one way to mitigate the extreme dense fog conditions. Results of analysis show that if we increase transmitted power then the received power, data rate, SNR and link margins are improved. Our results help us to decide the improvement level up to a limit to achieve
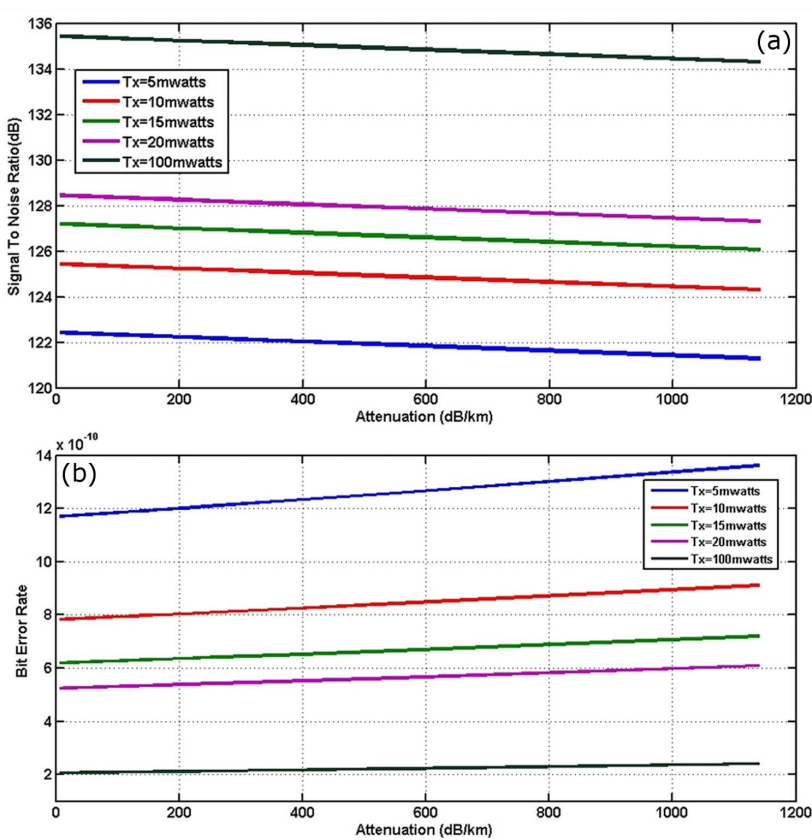

Fig. 2. (a) Illustration of attenuation against SNR, (b) illustration of attenuation against BER.

required data rate. Here, we have estimated above mentioned parameters at five different transmitted powers of $5,10,15,20$, and $100 \mathrm{~mW}$ for a link distance of $1 \mathrm{~km}$. At attenuation level of $38.99 \mathrm{~dB} / \mathrm{km}$, data rates of 2.82 , $5.87,7.98,9.74$ and 23.1 Gbps are achieved respectively for mentioned different levels of transmit power. The signal-to-noise ratio is $122.41,125.46,127.18,128.43$, and 135.42 , respectively. Estimated bit error rate for these respective transmitted powers are $1.18 \times 10^{-9}, 7.88 \times 10^{-10}$, $6.24 \times 10^{-10}, 5.28 \times 10^{-10}$ and $2.09 \times 10^{-10}$. We have also observed that fog events occur in the months of December, January, and February in Pakistan and the performance of FSO links is affected for less than a month in a span of a year.

\section{References}

[1] S.A. Al-Gailani, A.B. Mohammad, R.Q. Shaddad, Int. Conf. Phot. Proc. 2330-5665, 121 (2012).

[2] A. Mahdy, J.S. Deogun, J. IEEE Proc. 1525-3511, 2399 (2004).

[3] G. Nykolak, P.F. Szajowski, G. Tourgee, H. Presby, Electron. Lett. 35, 578 (1999).

[4] A.K. Majumdar, J.C. Ricklin, Free-Space Laser Communications, Springer, New York (NY) 2008.

[5] O. Bouchet, H. Sizun, C. Boisrobert, F. de Fornel, P. Favennec, Opt. Commun. ISTE, 2006.

[6] P.W. Kruse, L.D. McGlauchlin, R.B. McQuistan, J. Sci. 137, 123 (1962).

[7] I.I. Kim, B. McArthur, E.J. Korevaar, Opt. Eng. 4214, 26 (2001).

[8] M. Al-Naboulsi, H. Sizun, F. de Fornel, Opt. Eng. 43, 319 (2004).

[9] D. Kedar, S. Arnon, Appl. Opt. 42, 4946 (2003). 